\title{
Low-Frequency Vibrational Spectroscopy: A Powerful Tool for Revealing Crystalline Magnetic Structures
}

\author{
Zihui Song, ${ }^{\dagger, \|}$ Xudong Liu, ${ }^{\ddagger}, \|$ Anish Ochani,, Suling Shen, ${ }^{\S}$ Qiqi Li, ${ }^{\S}$ Yiwen Sun, ${ }^{*, \S}$ and Michael \\ T. Ruggiero*,,$\perp$ \\ $\dagger$ Materials Science Program, University of Vermont, Burlington, VT 05405 USA \\ $\ddagger$ National-Regional Key Technology Engineering Laboratory for Medical Ultrasound, Guangdong, Key \\ Laboratory for Biomedical Measurements and Ultrasound Imaging, Department of Biomedical Engineering, \\ School of Medicine, Shenzhen University, Shenzhen 518060, China \\ -Department of Chemistry, SUNY College at Old Westbury, Old Westbury, NY 11568, USA \\ $\S$ National-Regional Key Technology Engineering Laboratory for Medical Ultrasound, Guangdong, Key \\ Laboratory for Biomedical Measurements and Ultrasound Imaging, Department of Biomedical Engineering, \\ School of Medicine, Shenzhen University, Shenzhen 518060, China \\ \|Equal Contribution \\ $\perp$ Department of Chemistry, 82 University Place, University of Vermont, Burlington, VT, 05405 USA \\ Received June 25, 2021; E-mail: ywsun@szu.edu.cn; Michael.Ruggiero@uvm.edu
}

\begin{abstract}
:
The magnetic configuration of crystalline solids can have a profound impact on material properties, ranging from subtle structural effects to macroscopic phenomena. With the increased adoption of magnetic crystals for advanced applications, there is a significant need for tools that can characterize and predict the nature of magnetic ordering in solids. While there certainly exist techniques for acquiring such insight, those methods can be plagued by constraints (such as requiring certain elements), ultimately limiting their utility. Here, the strong-dependence of low-frequency (terahertz) vibrational dynamics on weak and long-range forces in crystals is leveraged to determine the spin state of iron phosphate - a promising material for cathodes in lithium ion batteries. We highlight how terahertz time-domain spectroscopy - coupled with quantum mechanical simulations - can discern between various spin configurations in $\mathrm{FePO}_{4}$. Furthermore, the results of this work unambiguously show that the well-accepted space group symmetry for $\mathrm{FePO}_{4}$ is actually incorrect, and that low-frequency spectroscopic measurements provide a clearer picture of the correct structure over the gold-standard of X-ray diffraction. This work opens the door for characterizing, predicting, and interpreting crystalline magnetic ordering using low-frequency vibrational spectroscopy.
\end{abstract}

The magnetic configuration of solids is responsible for a rich and diverse set of physical properties, and magnetic materials are crucial for advanced applications, with some modern examples including photovoltaics, ${ }^{1,2}$ spintronics, ${ }^{3}$ and superconductivity. ${ }^{4}$ In addition to those applications that take direct advantage of magnetic ordering, there are countless related phenomena where atomic- and molecularspin arrangements play an influential role - ranging from phase transformations ${ }^{5,6}$ to thermal conductivity. ${ }^{7}$ Therefore, there clearly exists a need for methods that can fullycharacterize the magnetic configuration of solids. But, while there certainly exist methodologies for characterizing the magnetic arrangement in solids - such as electron paramagnetic resonance $(\mathrm{EPR})^{8}$ and Mössbauer spectroscopy - there are some limitations to these methods (e.g., only certain elements can be characterized by Mössbauer spectroscopy ${ }^{9}$ ) and alternative means of obtaining such insight is a longsought-after goal.

Low-frequency vibrational spectroscopy (terahertz/farIR) is a powerful tool for studying solid materials, as the vibrations that occur at terahertz frequencies originate from long-range and weak intermolecular interactions. ${ }^{10}$ This yields a method that is highly-sensitive to subtle differences in such forces, ${ }^{11,12}$ enabling detailed insight into related phenomena, such as the ability to clearly differentiate between crystalline polymorphs. ${ }^{13}$ Given that variations between different magnetic configurations will undoubtedly result in varying intermolecular interactions, terahertz vibrational methods should be able to register these differences, making it an indirect probe of magnetic structure in materials - regardless of what elements are present and without requiring large crystals. Coupling experimental terahertz spectroscopic data with ab initio density functional theory (DFT) simulations provides even greater insight into the atomic-level dynamics that shape the properties of materials. ${ }^{10}$ In addition, DFT simulations enable the prediction of properties that might not be readily accessible experimentally, and even allow for the creation of structures and magnetic configurations that haven't been discovered yet, which has direct implications in material dSupporting Informationgn and discovery applications.

In this work, Iron (III) phosphate $\left(\mathrm{FePO}_{4}\right)$ was chosen as a model system to explore the ability of terahertz timedomain spectroscopty (THz-TDS) to determine the magnetic configuration of crystals, owing to the detailed characterization data on this system available in the literature, including cryogenic Mössbauer spectroscopy and X-ray diffraction measurements. ${ }^{14-18}$ While not a new material, in recent years $\mathrm{FePO}_{4}$ has shown great promise as a cathode material in lithium ion batteries, ${ }^{19-21}$ and understanding its magnetic and electrical properties is central to further innovation. $\mathrm{FePO}_{4}$ is reported to crystallize in an $\alpha$-quartz type structure, and is known to undergo a ferromagnetic (FM) to antiferromagnetic (AFM) transition at a Néel temperature of around $25 \mathrm{~K} .{ }^{14,16}$ While the iron atoms in $\mathrm{FePO}_{4}$ are tetrahedrally coordinated, indicating that the iron is in the high-spin (HS) state, through the use of DFT simulations it is possible to explore all possible electronic configurations, and thus the low-spin (LS) state was also investigated here. 
Through a combined experimental approach consisting of powder X-ray diffraction (PXRD) and THz-TDS, and coupled to DFT simulations, a full accounting of the role of magnetic structure on other physical phenomena is uncovered. The data highlights that THz-TDS can indeed confirm (and by extension, help determine) the proper magnetic configuration of crystalline solids. Furthermore, the significant dependence of low-frequency vibrational dynamics on weak forces uncovers that the accepted crystal structure of $\mathrm{FePO}_{4}$ is most likely incorrect, suggesting that THz-TDS is more sensitive to subtle structural differences than X-ray diffraction methods, which are currently the arguable 'goldstandard' for structural determination.

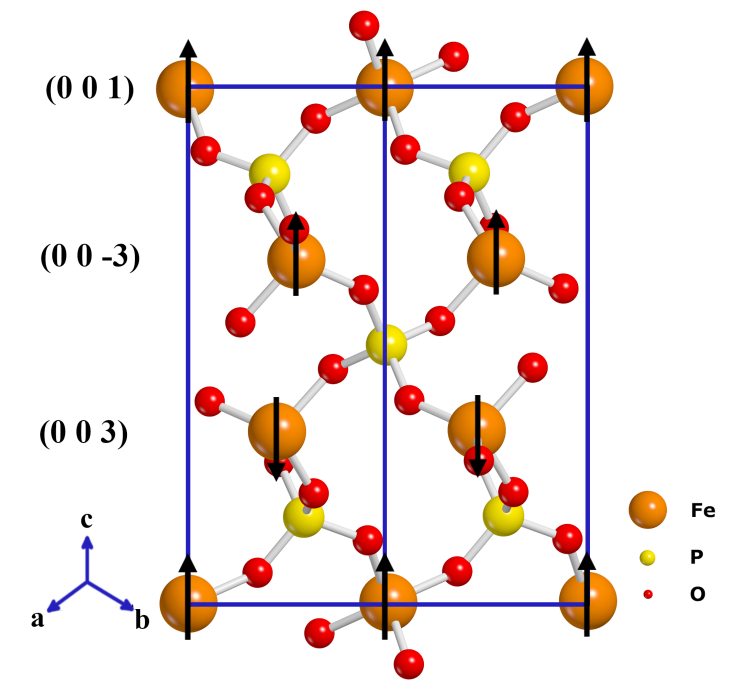

Figure 1. The crystal structure of $\mathrm{FePO}_{4}$, with the unit cell (in $P 3_{1} 21$ ) shown in blue. The black arrows represent the net spin state for each iron atom, shown here is the AFM arrangement with the net-spin on the iron atoms in the $\left(\begin{array}{llll}0 & 0 & 3\end{array}\right)$ plane set to $\beta$ (spin down).

The structure of $\mathrm{FePO}_{4}$ has previously been determined experimentally, and it is reported to exhibit an $\alpha$-quartz type structure in the $P 3_{1} 21$ space group (Figure 1). ${ }^{16}$ The structure consists of $\left[\mathrm{FeO}_{4}\right]^{-}$and $\left[\mathrm{PO}_{4}\right]^{+}$tetrahedra, which form an infinite network throughout the entirety of the crystal.

Quantum mechanical simulations were performed using the fully-periodic CRYstal17 software package, ${ }^{22}$ using the B3LYP ${ }^{23}$ functional and the Ahlrichs triple-zeta basis set with one polarization function (VTZP) ${ }^{24}$ for the iron atoms, and the triple-zeta $6-311 \mathrm{G}(2 \mathrm{~d}, 2 \mathrm{p})$ basis set ${ }^{25,26}$ for the nonmetals (additional simulation details available in the Supporting Information). One of the benefits of the CRYSTAL software package is that it is possible to fully exploit Bravais and space group symmetry. Thus, initially, closed-shell and high spin ferromagnetic (HSFM) simulations of $\mathrm{FePO}_{4}$ were performed within the $P 3_{1} 21$ space group, as any other magnetic configurations require a symmetry reduction due to the presence of only one unique iron atom in the unit cell (vide infra). It is important to note that the low-spin ferromagnetic configuration led to numerical instabilities in the simulation, and thus was neglected. The geometry of the structures were optimized, and all lattice vectors and atomic positions were allowed to relax, with no constraints other than the space-group symmetry of the solid - a method that has proven to be accurate on countless occasions in the past. ${ }^{27-34}$ The optimizations were successful, with an average error in the lattice parameters, compared to the experimental structure, of $0.592 \%$ and $1.438 \%$, for the closed-shell and HSFM simulations, respectively. The PXRD patterns for the two optimized structures were generated (Figure $\mathbf{2 C}$ and 2B, respectively) and compared to the experimental PXRD pattern (Figure 2A), and the agreement further highlights that the simulated structures were in apparent agreement with the experimental structure.

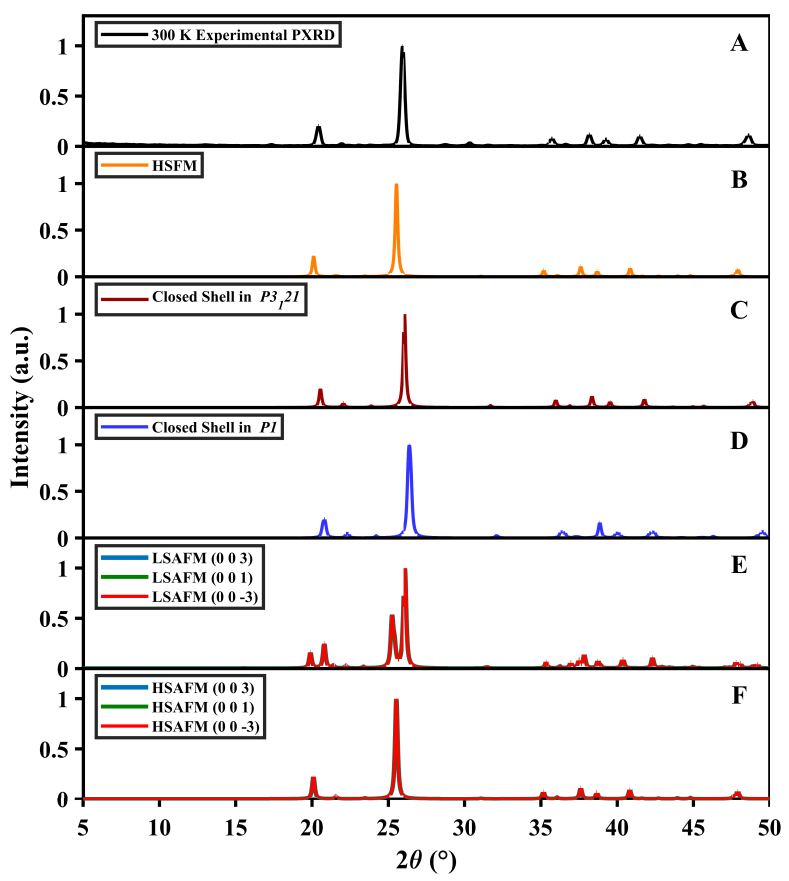

Figure 2. The $300 \mathrm{~K}$ experimental (A) and simulated $(\mathrm{B}-\mathrm{F})$ PXRD patterns of $\mathrm{FePO}_{4}$.

While vibrational spectroscopy is inherently a probe of inter-atomic forces, low-frequency vibrational spectroscopy is a powerful probe of three-dimensional structures, as the forces sampled at terahertz frequencies are often very-weak and long-range, meaning that they strongly depend on the bulk-packing structure. Thus, the simulated terahertz spectrum, when compared to the experimental one, can be used to not only understand weak forces, but also as a validation of the correct structure.

The terahertz spectra of dehydrated $\mathrm{FePO}_{4}$ were collected at $20 \mathrm{~K}$ and $300 \mathrm{~K}$ between $0.5-4.25 \mathrm{THz}$ using a commercial Toptica Teraflash Pro spectrometer (additional experimental details available in the Supporting Information). In the experimental spectra (Figure 3A), there were three clear absorption features, occurring at $1.22 \mathrm{THz}, 2.49 \mathrm{THz}$, and $3.66 \mathrm{THz}$ in the $300 \mathrm{~K}$ spectrum, with all three peaks exhibiting the characteristic sharpening upon cooling to $20 \mathrm{~K}$, while only the first and third peaks significantly blue-shift to 1.29 $\mathrm{THz}$ and $3.77 \mathrm{THz}$, respectively. Additionally, a broad absorption feature arises around $3.05 \mathrm{THz}$ upon cooling.

The optimized structures were used to initiate vibrational analyses - again maintaining space-group symmetry with the result of the HSFM shown in Figure 3B. In general, the agreement between the HSFM simulation and the room temperature terahertz spectrum is good. However, in the case of the closed-shell structure, the results of the vi- 


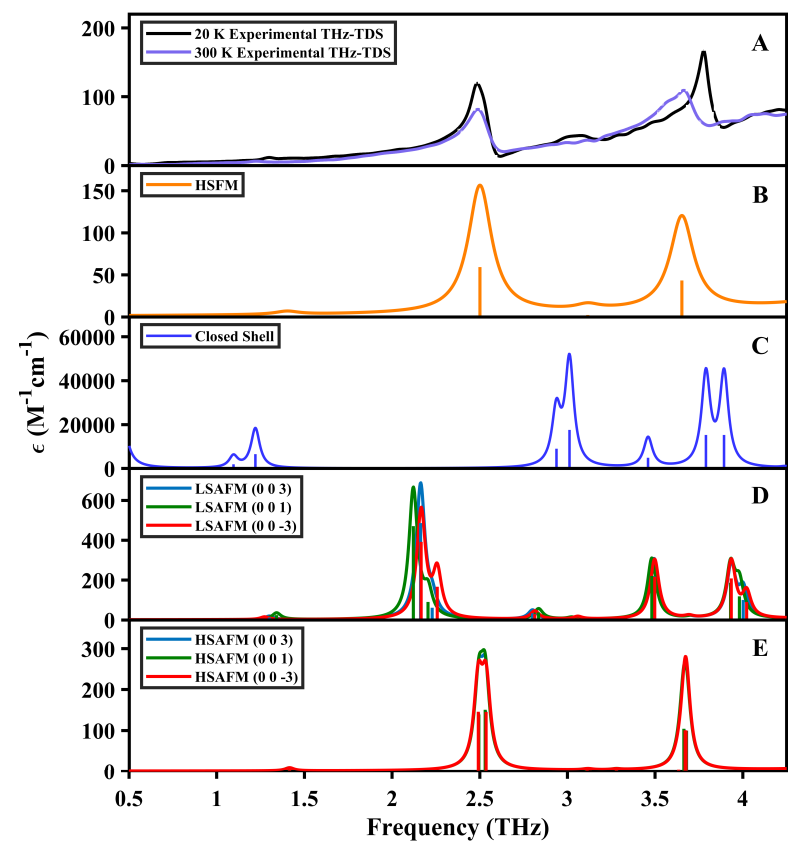

Figure 3. The experimental (A) and DFTpredicted (B-E) terahertz spectra of $\mathrm{FePO}_{4}$.

brational simulation yielded a negative frequency, indicating that the structure was not at the minimum on the potential energy surface (PES).

In order to further investigate the origin of this, the vibrational potential of the negative mode was determined by displacing the structure along the normal coordinate and determining the energy explicitly at each step. The result (see Supporting Information) indicates that the system was not at the global minimum on the PES. Thus, a new geometry optimization was performed starting from the the true minimum, with an important note being that the new structure did not exhibit any space-group symmetry, and thus the simulation was performed in the absence of symmetry (i.e., with a space group of $P 1$ ).

This optimization in the absence of symmetry gave comparable errors as the original $P 3_{1} 21$ optimization, with an average error of $1.665 \%$. The simulated PXRD pattern for the $P 1$ structure, shown in Figure 2D, was also in agreement with the experimental pattern. The subsequent vibrational frequency analysis did not yield any negative modes, confirming the success of the optimization.

The predicted closed-shell terahertz spectra and the experimental spectra are not in agreement (Figure $\mathbf{3 C}$ ), not only with respect to the vibrational frequencies, but also the intensities, which differ by 2 orders of magnitude, indicating that this was not the correct structure and/or magnetic configuration of $\mathrm{FePO}_{4}$.

While the room-temperature spin configuration is believed to be ferromagnetic, ${ }^{14}$ there is a reported FM to AFM transition at low-temperatures. ${ }^{14,15}$ Thus, both the low- and high-spin AFM configurations were studied in order to explore the influence of the spin configuration on the lowfrequency dynamics. The published space-group symmetry of the solid does not allow for the consideration of the AFM state, as there is only one symmetry-unique iron atom in the $P 3_{1} 21$ structure. Thus, in order to account for AFM behavior, the symmetry was initially reduced, resulting in structures with C2 space group symmetry.

Here, we have chosen to label the plane of atoms that are given $\beta$ spin (net negative spin), which assumes the other planes contain $\alpha$ spins (net positive spin). For this system, there are three possible configurations, $\beta$ in the $\left(\begin{array}{lll}0 & 0 & 3\end{array}\right)$, $\left(\begin{array}{lll}0 & 0 & 1\end{array}\right)$ or $\left(\begin{array}{lll}0 & 0 & -3\end{array}\right)$ planes. An example of the first case is shown in Figure 1. For this work, we have simulated all three possible magnetic arrangements leading to three possible configurations.

The first configuration tested was the low-spin antiferromagnetic (LSAFM) state. As done previously, the structure was first optimized, with a critical difference here being the necessary reduction of symmetry. The errors of the optimization were generally good, with errors of $1.150 \%, 2.480 \%$ and $1.152 \%$ for the three possible LSAFM configurations, compared to the experimental lattice vectors.

Following the geometry optimization, frequency analyses were performed, and again, the result produced a negative frequency for all three structures. The vibrational potential for that mode was again scanned, in the same manner as the closed-shell system. The new lattice and atomic coordinates were generated from the minimum point, see Supporting Information. The structure generated again resulted in no space-group symmetry $(P 1)$, and was subsequently reoptimized, followed by a frequency analysis.

While the resultant frequency calculations produced no negative frequencies, neither the simulated PXRD (Figure 2E) nor the simulated terahertz spectra (Figure 3D) are in agreement with the experimental results. However, while the PXRD patterns are essentially identical between the different LSAFM configurations studied, the simulated terahertz spectra vary. This highlights how sensitive terahertz dynamics are to subtle differences in weak intermolecular forces, which are clearly significantly influenced by the chosen magnetic configuration, and opens up the possibility for using THz-TDS to confirm and predict the nature of magnetic ordering in complex crystals, providing a powerful complement to existing methods.

The high-spin antiferromagnetic (HSAFM) models were generated in the same manner as the LSAFM structures, with three possible configurations. As done previously, the symmetry was reduced in order to allow for the AFM configuration, resulting in a space group of C2. The errors of the optimization were generally good, with errors of $1.471 \%$, $1.467 \%$ and $1.470 \%$ for the three possible HSAFM configurations, respectively. The simulated PXRD patterns for all three structures were in excellent agreement with the experimental PXRD pattern, as shown in Figure 2F.

The vibrational frequency analyses were performed in the same manner as previously described, and the experimental terahertz and HSAFM simulated spectra were in excellent agreement, with the simulated spectra all being nearly indiscernible, as presented in Figure 3E. Here, both the frequency of the transitions, and the IR intensities, matched the experimental data, highlighting the accuracy of the theoretical model and the correct representation of weak forces and electronic density. It is important to note that while this structure utilized space-group symmetry (C2), for completeness, simulations were also performed in the absence of symmetry $(P 1)$, which did not change the results.

Interestingly, the HSAFM simulations predict two modes at the location of the two most intense terahertz absorption features occurring at $2.48 \mathrm{THz}$ and $3.77 \mathrm{THz}$ in the experimental spectrum. In the experimental spectrum, those 

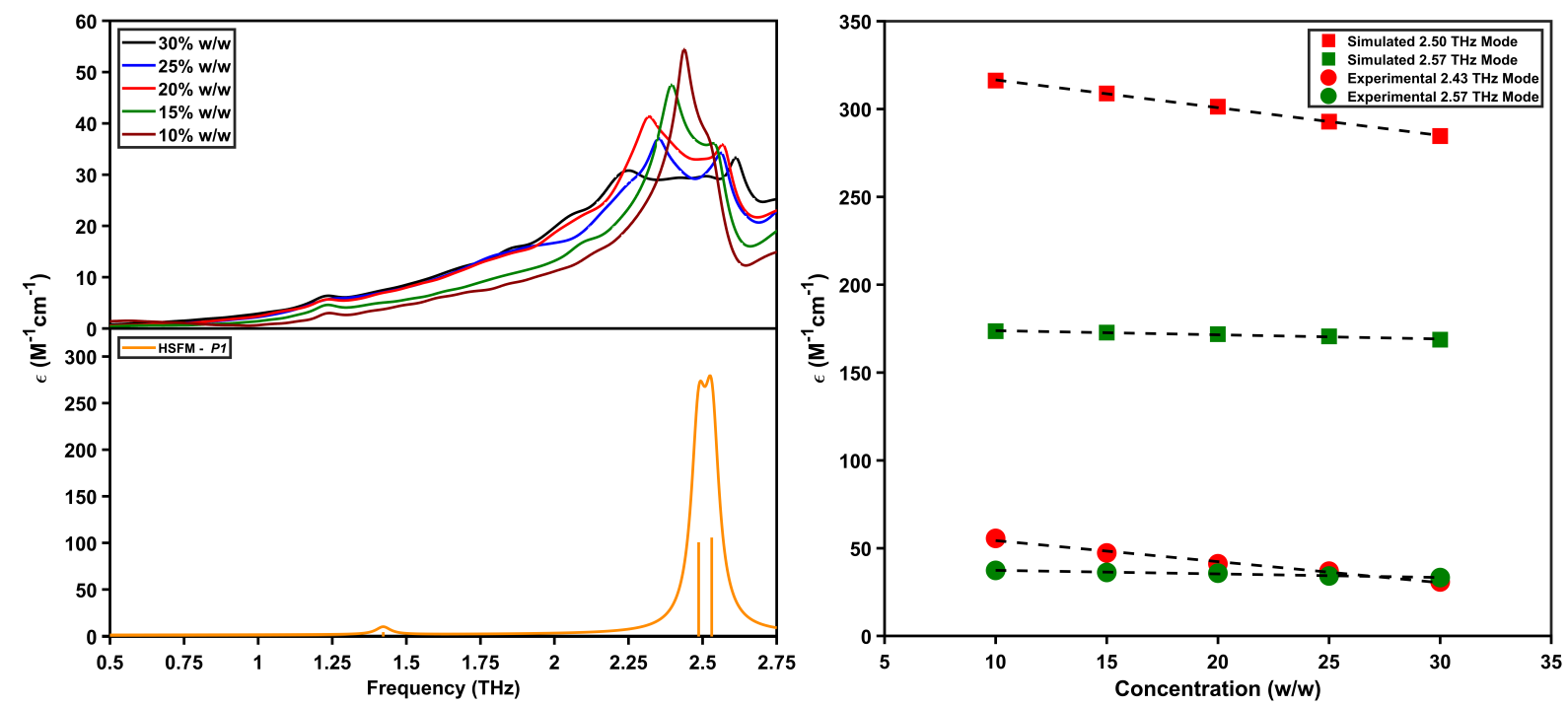

Figure 4. Experimental $(298 \mathrm{~K})$ variable concentration terahertz spectra (top left), predicted HSFM spectrum in the absence of symmetry (bottom left). The experimental peak intensities (circles) and Pdielecpredicted peak intensities (sqaures) are shown on the right.

peaks appear to be slightly asymmetric, indicating that they might arise from multiple transitions.

Surprisingly, the $298 \mathrm{~K}$ spectrum also suggests the presence of two discrete transitions, while the simulated HSFM spectrum in the published $P 3_{1} 21$ space group consisted of two degenerate modes. Close inspection highlights that the degeneracy arises due to the imposed $P 3_{1} 21$ symmetry, and upon re-optimizing the HSFM structure in $P 1$, that degeneracy is lifted again. The HSFM structure was also simulated in the $C 2$ space group, which did not change the calculated vibrational frequencies or intensities. In order to investigate this further, new experimental terahertz spectra were obtained with increased concentrations, from $10 \% \mathrm{w} / \mathrm{w}-30 \%$ w/w, with the results shown in Figure 4.

While terahertz absorption typically follows BeerLambert behavior, the measurements shown in Figure 4 (left panel) clearly do not - with both the frequencies and the intensities of the absorption features varying as the concentrations are increased, with the intensities of the terahertz modes near $2.5 \mathrm{THz}$ decreasing as concentration is increased. Such a result is clearly surprising, as it is exactly the opposite of what is expected with the Beer-Lambert Law (within the linear regime). It is important to note that at lower concentrations (below $10 \% \mathrm{w} / \mathrm{w}$ ), the absorption does obey the Beer-Lambert Law, indicating this these effects only become apparent at higher concentrations.

One possible explanation for this behavior is that there is a modification of the local electric field within the sample pellet, which was previously been demonstrated for ionic crystals with strong dipole moments. ${ }^{35}$ To investigate this, the PDielec package was used to post-process the simulated vibrational spectra to account for such behavior using effective medium theory, with the Maxwell-Garnett mixing rule. ${ }^{35}$ The results of these calculations are shown in Figure 4 (right panel), and it is clear the trends in the intensities between the experimental and theoretical data as a function of concentration are in excellent agreement. The magnitudes are not in agreement, but these experiments were performed at room temperature while the simulations assume an ef- fective temperature of $0 \mathrm{~K}$, which helps to account for this discrepancy. Additionally, the simulated intensities are actually integrated intensities, and different peak shapes (i.e. Gaussian, Lorentzian, and so on) would result in different peak heights, further adding to possible disagreement when only comparing the maximum intensity of an absorption feature.

Overall, the combination of terahertz THz-TDS and DFT simulations is powerful for determining and characterizing the magnetic structure of crystalline solids, with the results here going further to strongly support that the space group of $\mathrm{FePO}_{4}$ is actually C2, instead of the reported $P 3_{1} 21$. While an effective breaking of symmetry due to imposing magnetic ordering would not necessarily imply that the experimentally-assigned space group is incorrect, the breaking of vibrational mode degeneracy does, in fact, strongly support that the symmetry of $\mathrm{FePO}_{4}$ is not merely broken due to spin considerations, but also has its origins in the structure itself. The differences between the $P 3_{1} 21$, and $C_{2}$ structures are incredibly subtle, and the PXRD (and even single-crystal XRD) data are clearly unable to discern between these two structures. Yet, due to the dependence of terahertz vibrations on weak, long-range forces, subtle structural differences can have a pronounced impact on the lowfrequency vibrational spectra, thus highlighting that lowfrequency vibrational spectroscopy is a powerful tool for elucidating structures and magnetic configuration in solids. This work opens the door to utilizing low-frequency vibrational spectroscopy for identifying the magnetic configuration and structures of materials.

Acknowledgement MTR thanks the American Chemical Society Petroleum Research Fund (61794-DNI10), and the University of Vermont for support. XL, SS, QL, and YS gratefully acknowledge partial financial support for this work from the National Natural Science Foundation of China (Award Numbers 61975135 and 61805148), International Cooperation and Exchanges NSFC (Award Number 61911530218), Shenzhen International Scientific and Technological Cooperation Project (Award Number 
GJHZ20190822095407131) and Natural Science Foundation of Guangdong Province (Award Number 2019A1515010869), Guangdong Medical Science and Technology Research Fund (Award Number A2020401), Shenzhen University New Researcher Startup Funding (Award Number 2019134, RC00058).

\section{Supporting Information}

Experimental and theoretical methods, normal mode potential energy scans, and simulated crystal structures. This data is available on the web at ((link to be added))

\section{References}

(1) Phillips, P. From Insulator to Superconductor. Nature 2000, 406, 687-688.

(2) Ning, W. et al. Magnetizing Lead-Free Halide Double Perovskites. Sci Adv 2020, 6

(3) Puebla, J.; Kim, J.; Kondou, K.; Otani, Y. Spintronic Devices for Energy-Efficient Data Storage and Energy Harvesting. Commun. Mater. 2020, $1,24$.

(4) Diesch, S.; Machon, P.; Wolz, M.; Sürgers, C.; Beckmann, D. Belzig, W.; Scheer, E. Creation of equal-spin triplet superconductivity at the $\mathrm{Al} / \mathrm{EuS}$ interface. Nat. Commun. 2018, 9.

(5) Takahashi, K.; Okai, M.; Mochida, T.; Sakurai, T.; Ohta, H.; Yamamoto, T.; Einaga, Y.; Shiota, Y.; Yoshizawa, K.; Konaka, H.; Sasaki, A. Contribution of Coulomb Interactions to a Two-Step Crystal Structure Phase Transformation Coupled with a Significant Change in Spin Crossover Behavior for a Series of Charged FeIIComplexes from 2, 6-Bis(2-methylthiazol-4 yl)pyridine. Inorg. Chem. 2018, 57, 1277-1287.

(6) Sung, R. C. W.; McGarvey, B. R. A Study of the Spin-State Transition and Phase Transformation in [Fe(bpp)2][CF3SO3|2.H2O and [Fe(bpp)2][BF4]2 Using Mn2+ Electron Spin Resonance. Inorg. Chem. 1999, 38, 3644-3650.

(7) Jenčič, B.; Prelovšek, P. Spin and thermal conductivity in a classical disordered spin chain. Phys. Rev. B 2015, 92.

(8) Weil, J. A. A review of electron spin spectroscopy and its application to the study of paramagnetic defects in crystalline quartz. Phys. Chem. Miner. 1984, 10, 149-165.

(9) Kuzmann, E.; Nagy, S.; Vértes, A. Critical review of analytical applications of Mössbauer spectroscopy illustrated by mineralogical and geological examples (IUPAC Technical Report). Pure Appl. Chem. 2003, 75, 801-858.

(10) Ruggiero, M. T. Invited Review: Modern Methods for Accurately Simulating the Terahertz Spectra of Solids. J. Infrared Millim. Terahertz Waves 2020, 41, 491-528.

(11) Ruggiero, M. T.; Gooch, J.; Zubieta, J.; Korter, T. M. Evaluation of Range-Corrected Density Functionals for the Simulation of Pyridinium-Containing Molecular Crystals. J. Phys. Chem. A 2016, 120, 939-947.

(12) Zaczek, A. J.; Catalano, L.; Naumov, P.; Korter, T. M. Mapping the polymorphic transformation gateway vibration in crystalline 1, 2, 4, 5-tetrabromobenzene. Chem. Sci. 2019, 10, 1332-1341.

(13) Zeitler, J. A.; Taday, P. F.; Newnham, D. A.; Pepper, M.; Gordon, K. C.; Rades, T. Terahertz pulsed spectroscopy and imaging in the pharmaceutical setting - a review. J. Pharm. Pharmacol. 2007, 59, 209-223.

(14) Grandjean, F.; Long, G. J. Mössbauer Spectral Study of the Low-Temperature Electronic and Magnetic Properties of $\alpha-\mathrm{FePO}_{4}$ and the Mixed Valence Iron(II/III) Phosphate $\mathrm{SrFe}_{3}\left(\mathrm{PO}_{4}\right)_{3}$. Inorg. Chem. 2019, 58, 13314-13322.

(15) Battle, P. D.; Cheetham, A. K.; Gleitzer, C.; Harrison, W. T. A.; Long, G. J.; Longworth, G. A novel Magnetic Phase Transition in Anhydrous Iron (III) Phosphate, $\mathrm{FePO}_{4}$. J. Phys. C: Solid State Phys. 1982, 15, L919-L924.

(16) $\mathrm{Ng}, \mathrm{H}$. N.; Calvo, C. Refinement of the Crystal Structure of the Low-quartz Modification of Ferric Phosphate. Can. J. Chem. $\mathbf{1 9 7 5}, 53,2064-2067$

(17) Bhalerao, G. M.; Hermet P.; Haines, J.; Cambon, O. Keen, D. A.; Tucker, M. G.; Buixaderas, E.; Simon, P. Dynamic Disorder and the $\alpha-\beta$ Phase Transition in Quartz-Type $\mathrm{FePO}_{4}$ at High Temperature Investigated by Total Neutron Scattering, Raman Spectroscopy, and Density Functional Theory. Phys. Rev. B 2012, 86, 134104

(18) Smirnov, M.; Mazhenov, N.; Aliouane, N.; Saint-Grégoire, P. Novel Features of the $\alpha-\beta$ Phase Transition in Quartz-Type $\mathrm{FePO}_{4}$ as Evidenced by X-ray Diffraction and Lattice Dynamics. J. Phys.: Condens. Matter 2010, 22, 225403.

(19) Hassoun, J.; Bonaccorso, F.; Agostini, M.; Angelucci, M.; Betti, M. G.; Cingolani, R.; Gemmi, M.; Mariani, C.; Panero, S.; Pellegrini, V.; Scrosati, B. An Advanced Lithium-Ion Battery Based on a Graphene Anode and a Lithium Iron Phosphate Cathode. Nano Lett. 2014, 14, 4901-4906.

(20) Marongiu, A.; Nußbaum, F. G. W.; Waag, W.; Garmendia, M.; Sauer, D. U. Comprehensive study of the influence of aging on the hysteresis behavior of a lithium iron phosphate cathode- based lithium ion battery - An experimental investigation of the hysteresis. Appl. Energy 2016, 171, 629-645.

(21) Yang, S.; Zavalij, P. Y.; Whittingham, M. S. Hydrothermal synthesis of lithium iron phosphate cathodes. Electrochem. commun. 2001, 3, 505-508.

(22) Dovesi, R.; Erba, A.; Orlando, R.; Zicovich-Wilson, C. M.; Civalleri, B.; Maschio, L.; Rérat, M.; Casassa, S.; Baima, J.; Salustro, S.; Kirtman, B. Quantum-Mechanical Condensed Matter Simulations with CRYSTAL. WIREs Comput Mol Sci 2018, 8, e1360.

(23) Becke, A. D. Density-functional thermochemistry. III. The role of exact exchange. Int $J$. Chem Phys, 1993, 98, 5648-5652.

(24) Schäfer, A.; Horn, H.; Ahlrichs, R. Fully Optimized Contracted Gaussian Basis Sets for Atoms Li to Kr. J. Chem. Phys. 1992, 97, 2571-2577

(25) McLean, A. D.; Chandler, G. S. Contracted Gaussian Basis Sets for Molecular Calculations. I. Second Row Atoms, $\mathrm{Z}=11-18 . J$. Chem. Phys. 1980, 72, 5639-5648.

(26) Krishnan, R.; Binkley, J. S.; Seeger, R.; Pople, J. A. SelfConsistent Molecular Orbital Methods. XX. A Basis Set for Correlated Wave Functions. J. Chem. Phys. 1980, 72, 650-654.

(27) King, M. D.; Korter, T. M. Effect of Waters of Crystallization on Terahertz Spectra: Anhydrous Oxalic Acid and Its Dihydrate. J. Phys. Chem. A 2010, 114, 7127-7138.

(28) King, M. D.; Blanton, T. N.; Korter, T. M. Revealing the True Crystal Structure of L-Phenylalanine using Solid-State Density Functional Theory. Phys. Chem. Chem. Phys. 2012, 14, 11131116

(29) King, M. D.; Buchanan, W. D.; Korter, T. M. Application of London-type Dispersion Corrections to the Solid-State Density Functional Theory Simulation of the Terahertz Spectra of Crystalline Pharmaceuticals. Phys. Chem. Chem. Phys. 2011, 13, $4250-4259$.

(30) Ruggiero, M. T.; Bardon, T.; Strlič, M.; Taday, P. F.; Korter, T. M. The Role of Terahertz Polariton Absorption in the Characterization of Crystalline Iron Sulfate Hydrates. Phys. Chem. Chem. Phys. 2015, 17, 9326-9334.

(31) Ruggiero, M. T.; Erba, A.; Orlando, R.; Korter, T. M. Origins of Contrasting Copper Coordination Geometries in Crystalline Copper Sulfate Pentahydrate. Phys. Chem. Chem. Phys. 2015 17, 31023-31029.

(32) Ruggiero, M. T.; Korter, T. M. The Crucial Role of Water in Shaping Low-Barrier Hydrogen Bonds. Phys. Chem. Chem. Phys. 2016, 18, 5521-5528.

(33) Ruggiero, M. T.; Zeitler, J. A. Resolving the Origins of Crystalline Anharmonicity Using Terahertz Time-Domain Spectroscopy and Ab Initio Simulations. J. Phys. Chem. B 2016 $120,11733-11739$.

(34) Ruggiero, M. T.; Gooch, J.; Zubieta, J.; Korter, T. M. Evaluation of Range-Corrected Density Functionals for the Simulation of Pyridinium-Containing Molecular Crystals. J. Phys. Chem. A 2016, 120, 939-947.

(35) Kendrick, J.; Burnett, A. D. PDielec: The calculation of infrared and terahertz absorption for powdered crystals. J. Comput. Chem 2016, 37, 1491-1504. 